\title{
Distinct Role of von Willebrand Factor Triplet Bands in Glycoprotein Ib-Dependent Platelet Adhesion and Thrombus Formation under Flow
}

Citation for published version (APA):

Fuchs, B., de Witt, S., Solecka, B. A., Kroening, M., Obser, T., Cosemans, J. M. E. M., Schneppenheim, R., Heemskerk, J. W. M., \& Kannicht, C. (2013). Distinct Role of von Willebrand Factor Triplet Bands in Glycoprotein lb-Dependent Platelet Adhesion and Thrombus Formation under Flow. Seminars in Thrombosis and Hemostasis, 39(3), 306-314. https://doi.org/10.1055/s-0032-1328971

Document status and date:

Published: 01/04/2013

DOI:

10.1055/s-0032-1328971

Document Version:

Publisher's PDF, also known as Version of record

Document license:

Taverne

Please check the document version of this publication:

- A submitted manuscript is the version of the article upon submission and before peer-review. There can be important differences between the submitted version and the official published version of record.

People interested in the research are advised to contact the author for the final version of the publication, or visit the DOI to the publisher's website.

- The final author version and the galley proof are versions of the publication after peer review.

- The final published version features the final layout of the paper including the volume, issue and page numbers.

Link to publication

\footnotetext{
General rights rights.

- You may freely distribute the URL identifying the publication in the public portal. please follow below link for the End User Agreement:

www.umlib.nl/taverne-license

Take down policy

If you believe that this document breaches copyright please contact us at:

repository@maastrichtuniversity.nl

providing details and we will investigate your claim.
}

Copyright and moral rights for the publications made accessible in the public portal are retained by the authors and/or other copyright owners and it is a condition of accessing publications that users recognise and abide by the legal requirements associated with these

- Users may download and print one copy of any publication from the public portal for the purpose of private study or research.

- You may not further distribute the material or use it for any profit-making activity or commercial gain

If the publication is distributed under the terms of Article 25fa of the Dutch Copyright Act, indicated by the "Taverne" license above, 


\title{
Distinct Role of von Willebrand Factor Triplet Bands in Glycoprotein Ib-Dependent Platelet Adhesion and Thrombus Formation under Flow
}

\author{
Birte Fuchs, PhD ${ }^{1}$ Susanne de Witt, MSc ${ }^{2}$ Barbara A. Solecka, MSc ${ }^{1}$ Mario Kröning ${ }^{1}$ \\ Tobias Obser ${ }^{3}$ Judith M. E. M. Cosemans, $\mathrm{PhD}^{2}$ Reinhard Schneppenheim, MD, PhD ${ }^{3}$ \\ Johan W. M. Heemskerk, PhD ${ }^{2}$ Christoph Kannicht, $\mathrm{PhD}^{1}$
}

\author{
${ }^{1}$ Octapharma R\&D, Department of Molecular Biochemistry Berlin, \\ Berlin, Germany \\ 2 Department of Biochemistry, Cardiovascular Research Institute \\ Maastricht, Maastricht University, Maastricht, The Netherlands \\ ${ }^{3}$ Department of Pediatric Hematology and Oncology, University \\ Medical Center Hamburg-Eppendorf, Hamburg, Germany
}

\begin{abstract}
Address for correspondence Christoph Kannicht, PhD, Octapharma R\&D, Department of Molecular Biochemistry Berlin, Walther-NernstStr. 3, D-12489, Berlin, Germany

(e-mail: christoph.kannicht@octapharma.com).
\end{abstract}

\author{
Abstract \\ Keywords \\ - collagen \\ - flow \\ - glycoprotein lb \\ - von Willebrand factor \\ triplets \\ - platelet adhesion
}

Multimeric glycoprotein von Willebrand factor (VWF) exhibits a unique triplet structure of individual oligomers, resulting from ADAMTS-13 (a disintegrin and metalloproteinase with thrombospondin type 1 motifs 13) cleavage. The faster and slower migrating triplet bands of a given VWF multimer have one shorter or longer $\mathrm{N}$-terminal peptide sequence, respectively. Within this peptide sequence, the $A 1$ domain regulates interaction of VWF with platelet glycoprotein (GP)Ib. Therefore, platelet-adhesive properties of two VWF preparations with similar multimeric distribution but different triplet composition were investigated for differential functional activities. Preparation A was enriched in intermediate triplet bands, whereas preparation B predominantly contained larger triplet bands. Binding studies revealed that preparation A displayed a reduced affinity for recombinant GPIb but an unchanged affinity for collagen type III when compared to preparation B. Under high-shear flow conditions, preparation A was less active in recruiting platelets to collagen type III. Furthermore, when added to blood from patients with von Willebrand disease (VWD), defective thrombus formation was less restored. Thus, VWF forms lacking larger-size triplet bands appear to have a decreased potential to recruit platelets to collagen-bound VWF under arterial flow conditions. By implication, changes in triplet band distribution observed in patients with VWD may result in altered platelet adhesion at high-shear flow.
Hemostasis requires the combined action of blood platelets and vascular and plasmatic factors, with von Willebrand factor (VWF) having a key role. In blood, VWF circulates as a large glycoprotein (GP) consisting of multimers of heterogeneous sizes. At high-shear flow conditions, VWF mediates platelet adhesion via the glycoprotein Ib (GPIb)-V-IX complex, in particular by depositing to collagen fibres, at which it avidly binds. Collagen-bound VWF thus plays a critical role in platelet

published online February 3, 2013
Issue Theme Quality in Hemostasis and Thrombosis, Part II; Guest Editors, Giuseppe Lippi, MD, Mario Plebani, MD, and Emmanuel J. Favaloro, PhD, FFSC (RCPA). tethering, translocation, and stable adhesion at arterial flow conditions above a critical shear rate of 500 to $1,000 \mathrm{~s}^{-1}$.,2 VWF binds to collagen types I and III via its A3 domain, whereas binding to type VI collagen has been attributed to VWF A1 domain. ${ }^{3-5}$ In addition, VWF is implicated in platelet GPIb-dependent procoagulant activity and fibrin formation, ${ }^{6}$ and it protects factor VIII from rapid proteolytic inactivation. $^{7}$
Copyright $(\odot) 2013$ by Thieme Medical Publishers, Inc., 333 Seventh Avenue, New York, NY 10001, USA.

Tel: +1(212) 584-4662.
DOI http://dx.doi.org/ 10.1055/s-0032-1328971. ISSN 0094-6176. 
It is well established that large VWF multimers, which are secreted from endothelial cells, are cleaved into smaller forms by the metalloproteinase ADAMTS-13 (a disintegrin and metalloproteinase with thrombospondin type 1 motifs 13 ). This protease cleaves the $\mathrm{Y}^{1605}-\mathrm{M}^{1606}$ peptidyl bond within the A2 domains of VWF multimers, thus generating smaller size multimers. ${ }^{8}$ Because of asymmetric cleavage in the VWF A2 domain, ADAMTS-13 degradation results in larger- and smaller-size monomeric forms of $176 \mathrm{kDa}$ (C-terminal peptide sequence) and $140 \mathrm{kDa}$ ( $\mathrm{N}$-terminal peptide sequence), respectively (-Fig. 1A). On agarose gels, these represent the two satellite bands flanking the major band of all VWF multimers, ${ }^{9,10}$ forming a so-called VWF triplet. The slower and faster migrating triplet bands thus either contain or lack one $140 \mathrm{kDa} \mathrm{N}$-terminal peptide sequence compared to the intermediate VWF triplet band, respectively. ${ }^{11}$ The variable part of individual VWF triplets resides in the $\mathrm{N}$-terminal protein part comprising the A1 domain, which contains binding sites for heparin, coagulation factor VIII, and GPIb and, hence, is highly relevant to VWF function.

Because the properties of individual VWF triplet bands have not been investigated so far, we aimed to separate normal plasma-derived VWF into subfractions with a similar multimeric composition but different triplet band patterns. Therefore, we made use of the property that the longer-chain, slower-migrating triplet bands of VWF multimers containing one additional N-terminal $140 \mathrm{kDa}$ peptide sequence more avidly bind to heparin. ${ }^{12}$ We used heparin affinity column

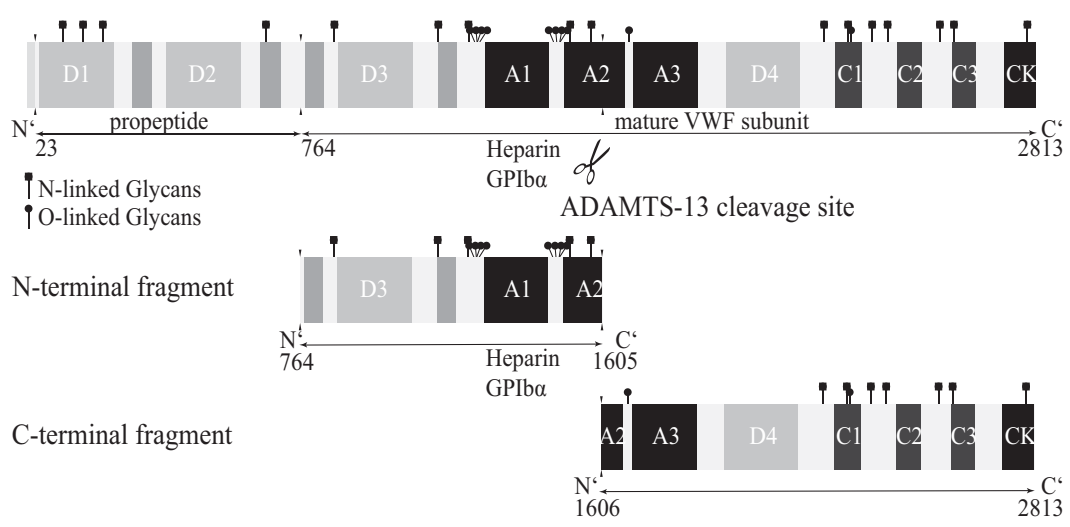

A

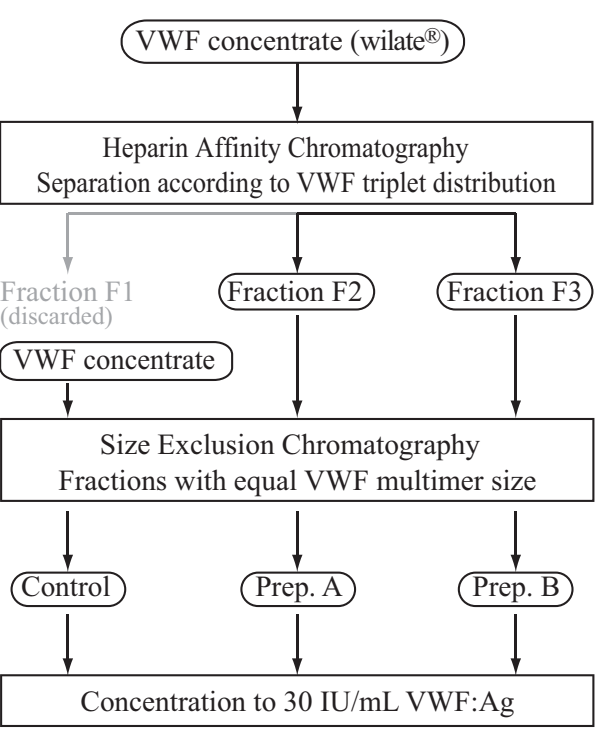

Fig. 1 Domain structure of VWF and flowchart to obtain preparations with different VWF triplet distribution. (A) VWF domains indicating binding sites for heparin and GPIb in the A1 domain. The propeptide of AA 23-763 after cleavage of the prepropeptide is depicted below the domain structure as well as the mature VWF protein starting with AA 764-2813 (numbering according to the preproprotein). Glycosylation is indicated as squares (N-linked glycans) and circles (O-linked glycans). ADAMTS-13 (a disintegrin and metalloproteinase with thrombospondin type 1 motifs 13 ) cleavage site in the A2 domain is indicated as well. This cleavage results in a $140 \mathrm{kDa}$-terminal fragment comprising the D3-A1 domains and part of the A2 domain with binding sites, for example to GPIb and heparin. Cleavage also results in a larger $176 \mathrm{kDa}$ C-terminal fragment, comprising part of the A2 domain as well as VWF domains A3D4C1C2C3CK. Information source: P04275, UniProtKB/Swiss-Prot database. (B) Flowchart to separate plasma-derived VWF into preparations with different triplet composition, but similar multimeric composition. Application of desalted VWF concentrate (wilate ${ }^{\circledR}$ ) on heparin affinity chromatography column results in three fractions, fraction F1 (discarded) and fractions F2 and F3 with distinct triplet composition. By using size exclusion chromatography, F2 and F3 are resolved in subfractions of similar molecular weight ranges and, hence, similar multimeric composition, giving preparations A and B, respectively. Desalted whole VWF concentrate is also subjected to size exclusion chromatography to obtain a control preparation with similar molecular weight ranges to preparations A and B. GPIb, glycoprotein Ib; VWF, von Willebrand factor. 
separation followed by size exclusion chromatography to obtain VWF preparations with a similar multimer profile but distinct pattern of triplet bands, resulting in different affinities to GPIb and different capabilities to promote GPIb-dependent platelet adhesion under high-shear flow conditions.

\section{Materials and Methods}

\section{Materials}

Plasma-derived VWF/factor VIII concentrate wilate ${ }^{\circledR}$ (lot no. A925A189) used for fractionation was from Octapharma (Vienna, Austria). VWF levels are given as antigen concentrations unless indicated otherwise. SHP was used as reference (Coagulation Control N, Technoclone, Vienna, Austria). Collagen type III from human placental villi was obtained from Biozol Diagnostica (Eching, Germany). F(ab')2 fragments of monoclonal antibody (Ab) 6B4 against the VWF-binding epitope on GPIb $\alpha$ was a kind gift of Prof. Dr. H. Deckmyn. ${ }^{13} \mathrm{H}-$ Phe-Pro-Arg chloromethyl ketone (PPACK) came from Calbiochem (La Jolla, CA).

\section{Isolation of VWF Preparations with Different Triplet Composition}

Plasma-derived VWF concentrate wilate ${ }^{\circledR}$ was desalted using a PD-10 column and eluted with $20 \mathrm{mM}$ Tris, pH 7.4 (GE Healthcare, Uppsala, Sweden). The VWF concentrate was then fractionated according to triplet structure by fast protein liquid chromatography (FPLC), employing a $5 \mathrm{~mL}$ HiTrap heparin HP affinity column (GE Healthcare) and stepwise gradient elution with 190,230 , and $500 \mathrm{mM} \mathrm{NaCl}$ in $20 \mathrm{mM}$ Tris, pH 7.4 (-Fig. 2A). Of the three fractions (F1-3), only F2 and F3, obtained at 230 and $500 \mathrm{mM} \mathrm{NaCl}$ elution, respectively, were used for further purification. The other fraction F1 was enriched in the faster-migrating triplet band but contained only small VWF multimers with low biologic activity (- Fig. 2A), ${ }^{14}$ and was hence discarded.

Each of the fractions F2 and F3 ( unfractionated wilate ${ }^{\circledR}$ (original sample $=$ control preparation) were differentially separated into subfractions by FPLC using a Sepharose CL-2B size exclusion column. Elution was performed with Tris-buffer, pH 7.4 (20 mM Tris, 0.02\% v/v Tween 20). Subfractions from this column for each starting sample (i.e., fractions F2 and F3, and control preparation) were then separately pooled to yield different VWF preparations with a similar multimeric distribution. This was done for the control preparation (exemplary shown in - Fig. 2B), as well as for fractions F2 and F3 (not shown but similar process to that shown for control preparation in - Fig 2B) to give preparations A and B, respectively (see - Fig 1B). Each separate column fraction pool was then concentrated using an Amicon Ultra-4 centrifugal filter device (100K; Millipore, Billarica, MA) at 2,000 $g$ to a VWF:Ag level of about $30 \mathrm{IU} / \mathrm{mL}$. Fraction analysis for VWF multimer distribution was by $1.2 \%$ agarose gel electrophoresis; and analysis for VWF triplet composition was by $1.6 \%$ agarose gel electrophoresis, followed by Western blotting. ${ }^{15}$ Densitometric analysis of blots was performed with Multi Gauge V3.2 software (Fujifilm, Düsseldorf, Germany).

VWF preparations were assessed for collagen binding using a VWF:CB ELISA (enzyme-linked immunosorbent assay) (TECHNOZYM vWF:CBA ELISA Kit, Technoclone, Vienna, Austria). VWF ristocetin cofactor activity (VWF:RCo) was determined using a BCS XP system (Siemens Healthcare,
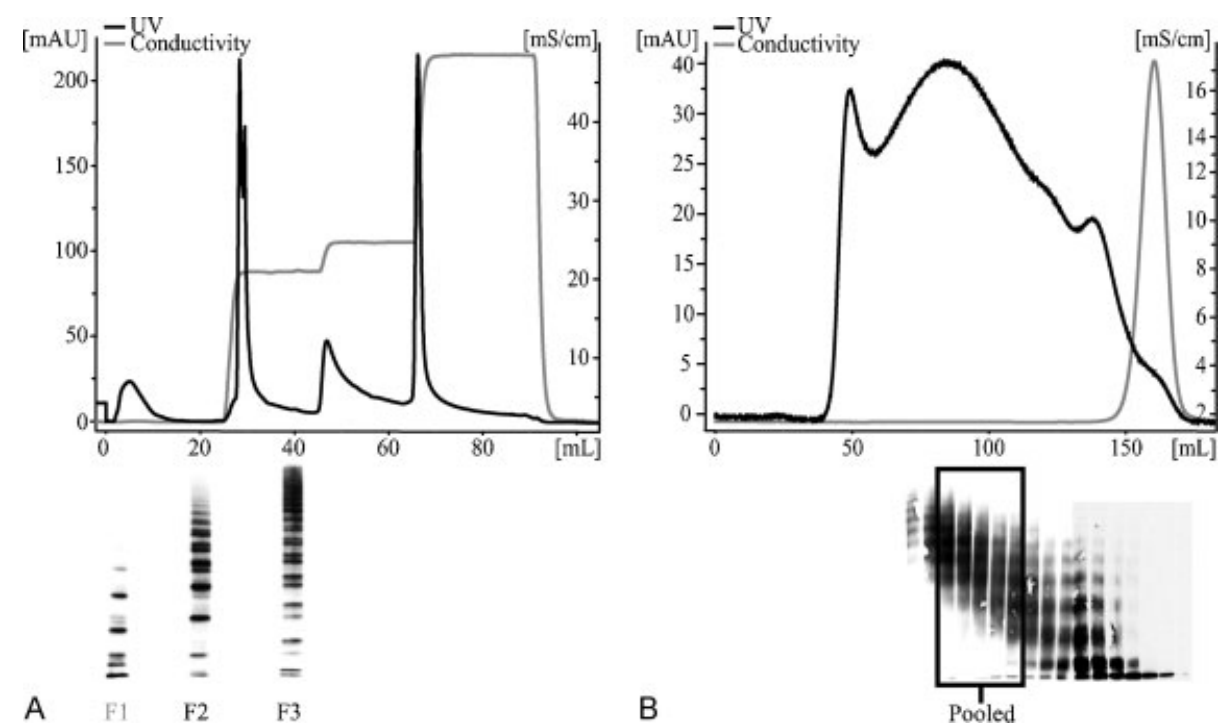

Fig. 2 Obtaining VWF preparations with different triplet forms by heparin affinity and size exclusion chromatography. (A) Plasma-derived VWF concentrate was applied to a heparin affinity column, and eluted step-wise with 190, 230, and $500 \mathrm{mM} \mathrm{NaCl}$ (gray line of eluent conductivity). Protein peaks were collected as fractions $1-3(\mathrm{~F} 1-3)$ and subjected to Western blotting (1.6\% agarose gels). Note differences in multimeric and triplet band composition of the fractions. F1 containing mostly small VWF multimers with little biological activity was discarded. F2 predominantly contained the medium to large VWF multimers with only intermediate triplet bands, whereas F3 consisted of more of the larger VWF multimers enriched in slower migrating triplet bands. (B) Size exclusion chromatography of unseparated VWF concentrate, resulting in initial elution of larger VWF multimers. Western blots of $1.2 \%$ agarose gels illustrate the multimeric sizes of individual fractions, and pooling of fractions to obtain the control VWF preparation. Note that preparations A and B were obtained using the same size exclusion chromatography as shown for the control in (B) but starting from fractions F2 and F3, respectively (not shown in this figure). VWF, von Willebrand factor. 
Eschborn, Germany); all values were calibrated against standard human plasma (SHP).

\section{ELISA Assays}

Antigen concentrations of VWF were determined with a sandwich ELISA with polyclonal anti-human VWF antibody (Ab) (A0082, Dako, Hamburg, Germany) for capturing, followed by staining with horseradish peroxidase (HRP)-conjugated anti-human VWF Ab (P0226, Dako). Sigma Fast OPD tablets were used as chromogenic substrate (order no. P9187; SigmaAldrich, Schwerte, Germany), color development was terminated with $1 \mathrm{M} \mathrm{HCl}$, and absorbance was measured at $492 \mathrm{~nm}$.

Binding of VWF fractions to platelet-derived GPIb was determined with an immunoassay employing recombinant, FLAGtagged GPIb $\alpha$ fragment with two gain-of-function mutations (G233V and M239V), enabling VWF binding in the absence of ristocetin. ${ }^{16-18}$ The rGPIb $\alpha$ fragment was immobilized via its FLAG tag in 96-well plates precoated with anti-FLAG Ab (F3165, Sigma-Aldrich). Bound VWF was measured using a polyclonal anti-human VWF Ab and an HRP-conjugated secondary $\mathrm{Ab}$ (A0082 and P0226, Dako) in combination with TMB substrate solution (T0565, SIGMA FAST OPD peroxidase substrate [P9187], Sigma Aldrich). The coloring reaction was terminated with $0.5 \mathrm{M} \mathrm{H}_{2} \mathrm{SO}_{4}$, and absorbance was read at $450 \mathrm{~nm}$.

\section{Surface Plasmon Resonance}

Binding of VWF preparations to collagen type III was determined by surface plasmon resonance (SPR) using a Biacore instrument (GE Healthcare), CM5 chips, and amine coupling chemistry, basically as described. ${ }^{19}$ In brief, collagen type III was immobilized on the chip surface at an amount of 800 to 1,000 response units (RU). VWF samples were injected at increasing concentrations $(0.2,0.4,1.3$, and $4 \mathrm{IU} / \mathrm{mL})$ in HBS-EP running buffer (3.4 mM EDTA, $10 \mathrm{mM} \mathrm{HEPES,} 150 \mathrm{mM} \mathrm{NaCl}, 0.005 \% \mathrm{v} / \mathrm{v}$ Tween 20; $\mathrm{pH} 7.4$ ) at a flow rate of $20 \mu \mathrm{L} / \mathrm{min}$. A chip with immobilized polyclonal rabbit anti-human collagen I-V Ab (PC169, Kamiya Biomedical, Seattle, WA) served as reference for unspecific binding. Chips were regenerated after each run. ${ }^{20}$

For determination of rGPIb binding kinetics by SPR, purified gain-of-function rGPIb $\alpha$ fragment carrying a His-tag was immobilized onto NTA-chips (GE Healthcare) at 1,000 RU. Samples of VWF were injected at increasing concentrations of $0.3,0.9,2.8$, and $8.4 \mathrm{IU} / \mathrm{mL}$. Analysis of all SPR binding curves was done with BiaEvaluation software (GE Healthcare), assuming Langmuir 1:1 binding kinetics and a molecular weight of the VWF monomer of $270 \mathrm{kDa}$.

\section{Platelet Adhesion and Thrombus Formation under Flow}

Leukocyte-depleted platelet concentrates collected by aphaeresis and red cell concentrates were obtained from Haema (Berlin, Germany). These concentrates were used to prepare washed platelets and red cells, respectively. Shearand VWF-dependent platelet adhesion to collagen type III using reconstituted blood was performed, as described elsewhere. ${ }^{21}$ Briefly, $2.5 \times 10^{8}$ platelets $/ \mathrm{mL}$ were labelled with 5chloromethylfluorescein diacetate (CMFDA, Invitrogen, Karlsruhe, Germany), and reconstituted with washed red cells (hematocrit of $40 \%$ ) and VWF preparations (1 IU/mL). Viscosity of the reconstituted blood samples was measured with an AMVn falling sphere viscometer (Anton Paar, Ostfildern, Germany) to ensure a dynamic viscosity of approximately $3 \mathrm{mPa}$.s. Samples were perfused at a shear rate of $1,700 \mathrm{~s}^{-1}$ through a flow chamber coated with collagen type III ( $\mu$-slide VI, Ibidi, Munich, Germany). Platelet adhesion was monitored in real time for up to 8 minutes. After perfusion and fixation, 24 randomly chosen images in the centre of the flow channel were recorded and analyzed using AxioVision 4.6 software (Carl Zeiss, Göttingen, Germany).

The VWF preparations were also tested in whole-blood perfusion assays, using a miniaturized parallel-plate flow chamber and equipment, as described before. ${ }^{22,23}$ Chamber surfaces were coated with collagen type III $(0.1 \mathrm{mg} / \mathrm{mL})$, and blocked with HEPES buffer, pH 7.45 plus 1\% BSA. Experiments were carried out with blood from healthy controls and from two patients with VWD. A patient with type 1 VWD had a VWF:RCo activity of $29 \%$. A patient with type 2A VWD had a VWF:RCo activity of $<10 \%$, a VWF antigen level of 25 to $35 \%$, and a factor VIII level of 30\%; multimer analysis revealed that the highest multimers were lacking, which is compatible with type $2 \mathrm{~A}$.

Blood was drawn into PPACK anticoagulant, as described previously. ${ }^{24}$ This direct thrombin active-site inhibitor achieves complete anticoagulation while maintaining physiologic concentrations of free $\mathrm{Mg}^{2+}$ and $\mathrm{Ca}^{2+}$ ions. Blood samples were spiked with vehicle or VWF preparations at final concentrations of $2 \mathrm{IU} / \mathrm{mL}$. The blood was then perfused through the flow chamber at a shear rate of $1,700 \mathrm{~s}^{-1}$ for 4 minutes. Four to five randomly chosen phase-contrast images were captured with a digital CCD camera at the end of perfusion. Images were analyzed as described earlier. Blood donors had given full informed consent, and not taken medication interfering with platelet function for at least 2 weeks.

\section{Statistical Analyses}

Significance of data was analyzed with GraphPad Prism version 5.01 (GraphPad Software, San Diego, CA). Normal distribution was confirmed using the Kolmogorov-Smirnov normality test. Differences between grouped data were compared with an unpaired Student $t$ test. Data from gels with VWF multimers and SPR analyses were compared by two-way analysis of variance, using Bonferroni posttesting to compare replicates.

\section{Results}

Separation of plasma-derived VWF by heparin affinity chromatography ( $\mathbf{- F i g . ~ 1 ) ~ r e s u l t e d ~ i n ~ t w o ~ f r a c t i o n s ~ ( F 2 ~ a n d ~ F 3 ) ~}$ with marked differences in triplet structure, but also to some extent in multimeric composition (-Fig. 2A). Each of the fractions, as well as the original VWF preparation (used as a control) was further separated by size exclusion chromatography, and subfractions were pooled to obtain preparations with equal multimeric distribution (process shown in - Fig. 2B exemplary for the control).

The resulting fractionated VWF pools, preparation A and B, as well as the (unfractionated) control preparation, were 


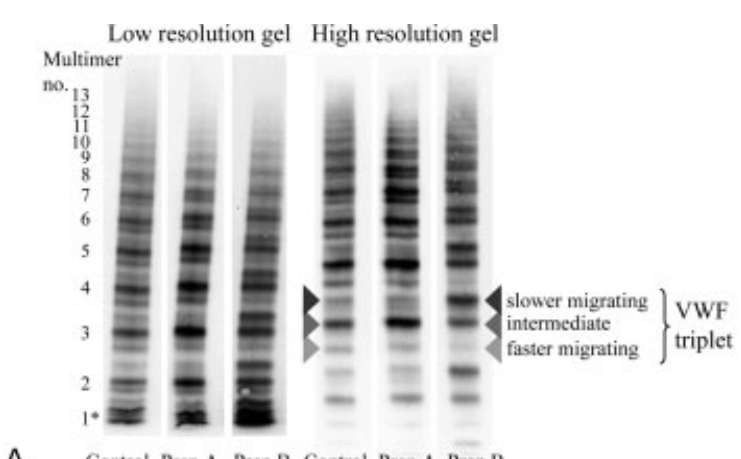

A

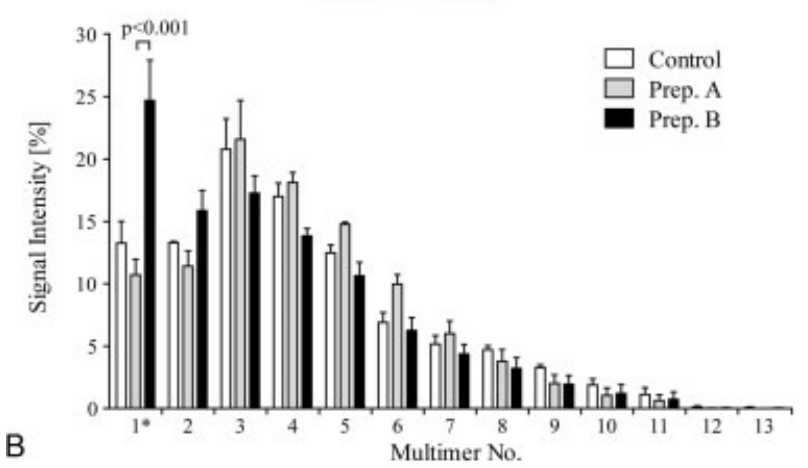

B

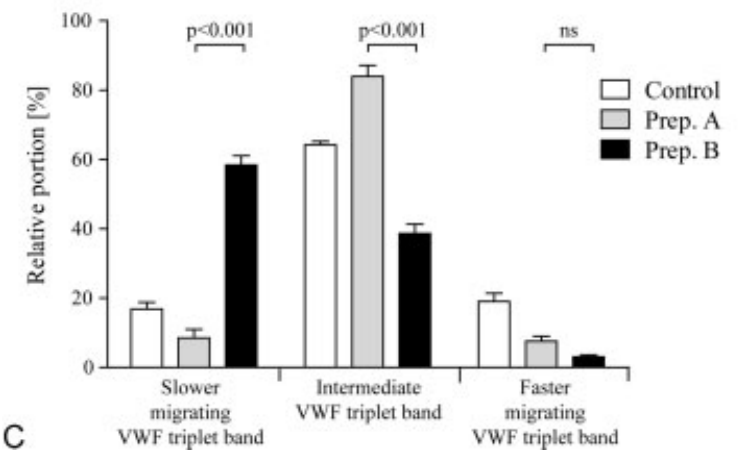

Fig. 3 Triplet and multimer composition of fractionated VWF preparations. Comparison of control preparation with normal triplet structure (VWF concentrate subjected to size exclusion chromatography), and preparations $A$ and $B$ (separated by heparin affinity chromatography and then size exclusion chromatography) analysed by agarose gel electrophoresis and Western blotting. (A) Representative Western blots. Left: low-resolution $1.2 \%$ agarose gels, showing multimer sizes from 1 mer to 13 mer ( ${ }^{*} 1$ mer band plus dye front). Right: high-resolution $1.6 \%$ agarose gels, showing triplet composition of multimers with faster, intermediate and slower migrating triplet bands (arrowheads). Note that preparations A and B, and the control preparation were similar in multimer composition (2-13mer). (B) Relative distribution of VWF 1-13mer multimers of control preparation and preparations A and B. (C) Proportions of each of the three triplet bands in control preparation and preparations $A$ and $B$. Mean $\pm \operatorname{SEM}(n=4)$. Note here the difference in triplet bands: Preparation $A$ was enriched in intermediate triplet bands, while preparation B predominantly contained larger triplet bands. SEM, standard error of mean; VWF, von Willebrand factor.

characterized by electrophoresis on low- and high-resolution agarose gels and Western blotting (-Fig. 3A). Densitometric analysis of blots showed that, as aimed for, preparations $A$ and $B$ were similar in multimer composition (2 to $13 \mathrm{mer}$ ), as was the control preparation ( $\mathbf{F}$ Fig. 3B, $p>0.05$ ). A significant difference in the preparations was only seen in the 1-mer composition, but this was not expected to influence subse- quent findings. Importantly, the various preparations displayed marked and significant differences with regard to triplet band composition ( $\mathbf{- F i g . 3 C}$ ). Preparation B was greatly enriched in the slower migrating triplet bands of essentially all multimers at the expense of intermediate triplet bands; in comparison, preparation A was greatly reduced in the slower migrating triplet bands and contained larger proportions of intermediate triplet bands ( $p<0.001$ ). Accordingly, the twocolumn separation had resulted in two VWF fractions with prominent differences in presence of larger-size triplet bands, but with similar multimeric composition.

The original VWF concentrate as well as the various preparations were concentrated to obtain solutions with the same VWF antigen concentrations, and further characterized using conventional VWF assays, namely VWF:CB and VWF:RCo. VWF:CB to VWF:Ag ratios were: $1.21 \pm 0.10$ (original sample), $0.91 \pm 0.02$ (control), $0.81 \pm 0.02$ (Prep. A), and $0.78 \pm 0.01$ (Prep. B); VWF:RCo to VWF:Ag ratios were $0.41 \pm 0.04$ (original sample), $0.37 \pm 0.01$ (control), $0.37 \pm 0.01$ (Prep. A), and $0.42 \pm 0.01$ (Prep. B). Hence, in comparison to the original VWD concentrate, the columnseparated preparations were slightly reduced in collagen binding and ristocetin cofactor activity, most likely due to partial retaining of the highest multimers on the heparin column (see following). Notably, preparation B had higher VWF:RCo, but similar VWF:CB compared to preparation A.

Using SPR, we analyzed binding properties of the three preparations to surface-immobilized collagen type III. Preparations A and B gave similar binding curves, when applied at increasing concentrations of 0.2 to $4 \mathrm{IU} / \mathrm{mL}$ (- Fig. 4A). Determination of the affinity constants, assuming conventional Langmuir kinetics, resulted in similar dissociation equilibrium constant $\left(K_{D}\right)$ values of 15 to $25 \mathrm{nM}$ ( - Fig. 4B), indicating no significant difference in binding affinity of the two preparations to collagen type III $(p=0.95)$. Both preparations displayed a tendency to diminished collagen binding in comparison to the unseparated control preparation.

Using a newly established method, a gain-of-function rGPIb fragment was immobilized to Biacore chips to investigate binding of the preparations to the platelet GPIb receptor. Binding curves were generated after injection of increasing concentrations of the VWF preparations (- Fig. 5A). Strikingly, the binding affinity of preparation $A$ in this case was significantly lower than of preparation B (-Fig. 5B, $p=0.0014$ ). This lower affinity was due to a slower association rate in combination with a faster dissociation rate. Furthermore, at equal antigen concentrations, the maximal binding was on average 30\% lower for preparation A than for preparation $B$, likely as a consequence of the lower affinity.

To confirm these differences in binding to GPIb, we also used an ELISA setup, where the same gain-of-function rGPIb fragment was immobilized on well plates. In this immunobased assay, preparation A again showed a lower binding to GPIb than preparation B (-Fig. 5C, $p<0.0001$ ), such in agreement with the results obtained by SPR.

For functional analyses, we then investigated the ability of the VWF preparations to support GPIb-dependent platelet adhesion to type III collagen at high shear flow conditions. In 


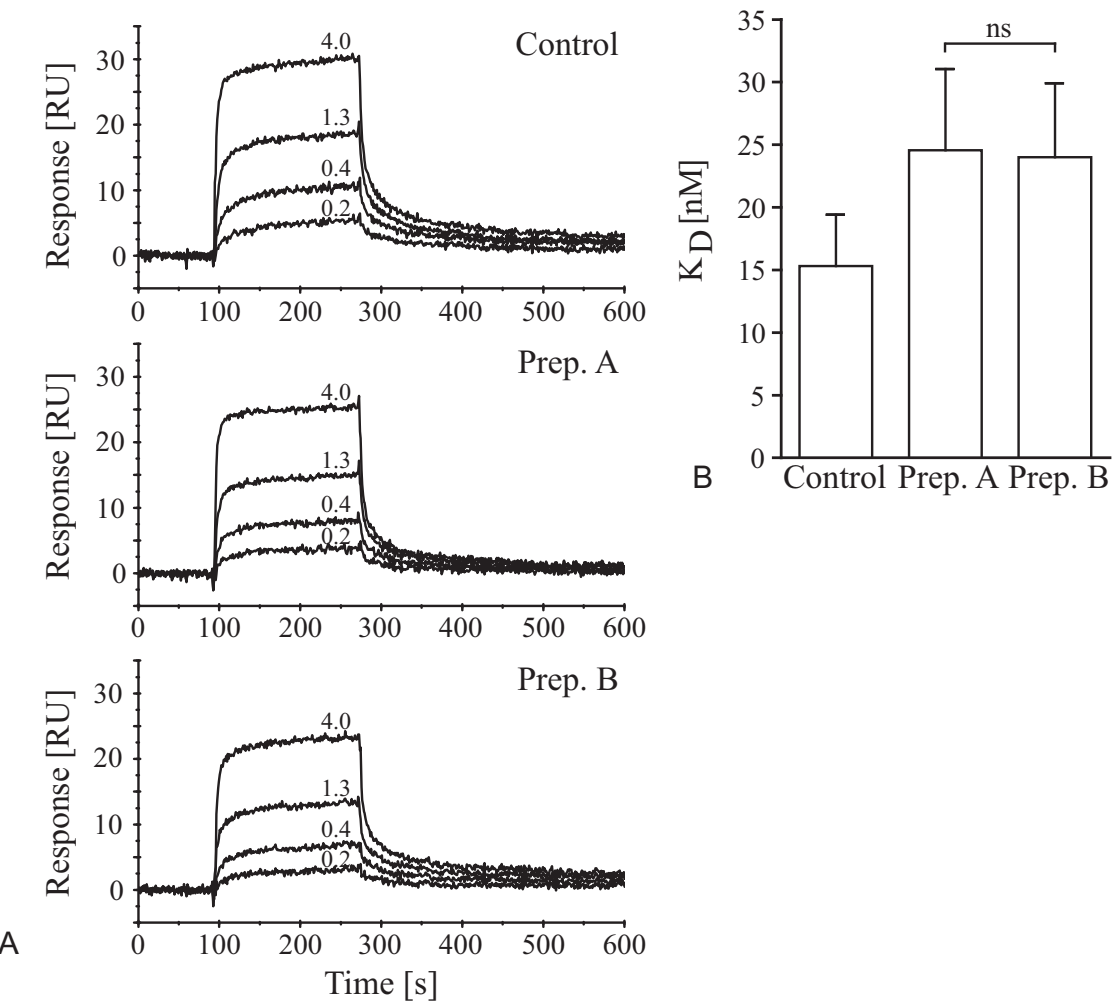

Fig. 4 Binding of VWF preparations to collagen. Binding kinetics of VWF preparations to collagen type III were established by surface plasmon resonance measurements. (A) Representative binding curves with increasing concentrations of $0.2,0.4,1.3$, and $4 \mathrm{IU} / \mathrm{mL}$ for control preparation and for preparations $A$ and $B$, as indicated. Binding is expressed as RU. (B) Calculated affinity constant values ( $\left.K_{D}\right)$ of the three VWF preparations. Mean \pm SEM $(n=6)$. RU, response units; SEM, standard error of mean; VWF, von Willebrand factor.

the first set of experiments, reconstituted blood samples containing platelets (CMFDA-labelled) and red blood cells were supplemented with the control VWF preparation or with preparation A or B at $1 \mathrm{IU} \mathrm{VWF} / \mathrm{mL}$. After perfusion over collagen, the coverage of adhered platelets was determined by fluorescence microscopy. In this flow assay, preparation A was significantly less active in supporting platelet adhesion than preparation B ( $\mathbf{- F i g . ~ 6 , ~} p<0.0001$ ). To demonstrate the importance of VWF-GPIb interaction, similar flow experiments were performed in the presence of $6 \mathrm{~B} 4 \mathrm{mAb}$, directed against the VWF binding site of GPIb. ${ }^{25}$ As indicated in - Fig. 6B, this antibody completely annulled the platelet-adhesive effect of the most active preparation B ( - Fig. $\mathbf{6 B}, p<0.0001$ ).

In a second range of experiments, we compared the effects of addition of a recombined F1-3 preparation with that of the original VWF concentrate. The combination of F1, F2, and F3 gave a multimeric distribution pattern similar to that of the VWF preparations A/B (Supplementary Fig. S1, online only). Addition of either preparation (2 IU VWF/mL, final) to the reconstituted blood resulted in a similar increase in platelet deposition on collagen as seen with the combination of preparations A and B (Supplementary Fig. S2, online only). This indicated that most of the biologic activity of VWF was retained in the preparations $\mathrm{A}$ and $\mathrm{B}$.

In addition, we performed perfusion studies with whole blood from VWD patients containing reduced baseline levels of VWF. Blood was thus obtained from a patient with VWD type 1
(29\% VWF:RCo activity) and a patient with VWD type 2A ( $<10 \%$ VWF:RCo activity). Samples of blood were spiked with the control preparation or with preparation A or B. After 4-minute perfusion at $1,700 \mathrm{~s}^{-1}$ shear rate, platelet deposition in thrombi was determined by enhanced contrast video microscopy. As shown in - Fig. 7, supplementation of all VWF preparations (control, A, or B) at $2 \mathrm{IU} / \mathrm{mL}$ VWF resulted in a significant increase in platelet deposition with both type 1 and type $2 \mathrm{~A}$ patient blood $(p<0.05)$. For the type $1 \mathrm{VWD}$ patient, no significant difference was seen between preparations $A$ and B. However, for the type 2A VWD patient, preparation A was significantly less effective than preparation $B$ in stimulating thrombus formation $(p=0.0133)$. Further control experiments showed that none of the preparations modified platelet deposition when added to blood from subjects with normal VWF levels (data not shown). Together, these findings suggest that VWF triplets lacking an additional $140 \mathrm{kDa} N$-terminal peptide sequence with the VWF A1 domain containing a GPIb binding site are decreased in binding affinity to platelet GPIb and are less active in supporting platelet thrombus formation on collagen type III under high-shear flow conditions.

\section{Discussion}

Distinct cleavage of VWF multimers in the A2 domain by the metalloproteinase ADAMTS- 13 results in VWF forms of various multimeric sizes, but with flanking satellite bands 

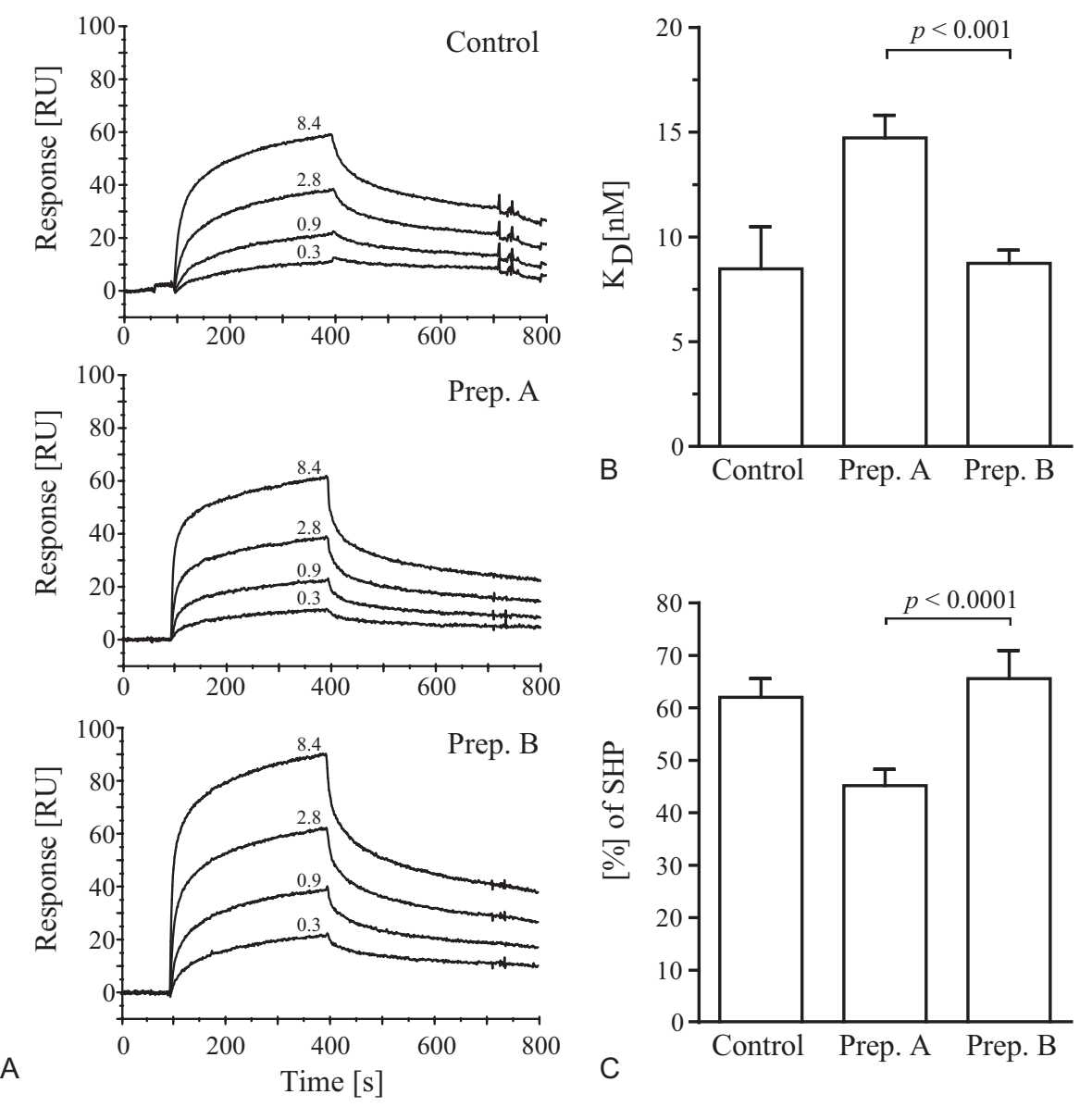

Fig. 5 Binding of VWF preparations to recombinant GPIb. Surface plasmon resonance measurements to assess binding of VWF preparations to a gain-of-function rGPIb fragment. (A) Representative binding curves, expressed as RU at increasing concentrations of $0.3,0.9,2.8$, and $8.4 \mathrm{IU} / \mathrm{mL}$ for control and preparations A and B. (B) Corresponding $K_{D}$ values. (C) Binding of VWF preparations to rGPIb obtained with ELISA. Data are expressed as percentage of binding seen in SHP. Mean \pm SEM $(n=5)$. ELISA, enzyme-linked immunosorbent assay; GPIb, glycoprotein Ib; RU, response units; SEM, standard error of mean; SHP, standard human plasma; VWF, von Willebrand factor.

(so-called triplet bands). ${ }^{10-12,26,27}$ The slower migrating triplet bands of VWF multimers thereby contain an additional 140 kDa N-terminal peptide fragment, whereas this $\mathrm{N}$ terminal part is missing in the faster migrating triplets when compared to the respective intermediate VWF triplet bands. ${ }^{11}$ Importantly, the N-terminal part of VWF monomers that is cleaved off by ADAMTS-13 proteolysis comprises the D1D2D3A1 domains and part of the A2 domain. This part is known to be involved in the binding of VWF to coagulation factor VIII, heparin, collagen, and platelet GPIb (reviewed by $^{28}$ ). The literature provides indirect evidence that distinct triplet bands of the VWF multimers may differ in functional properties, but so far this has not directly been shown. For instance, an altered VWF triplet composition is detected in type $2 \mathrm{~A}$ or $2 \mathrm{~B} \mathrm{VWD}$, that is, in forms of VWD that are characterized by altered VWF binding to platelets, likely as a consequence of changes in VWF proteolysis. ${ }^{29,30}$ In other disease forms, like type $2 \mathrm{M}$ VWD, satellite bands can even be absent, despite the presence of normal or even supra-normal multimers, which associates with defects in platelet-VWF interaction. $^{31}$

For this article, we have functionally characterized two VWF preparations separated by heparin affinity and size- exclusion chromatography, which are equal in VWF multimeric distribution patterns but markedly differ in triplet band composition. Binding studies using SPR- and ELISA-based approaches indicate that preparation A, lacking an extra Nterminal peptide sequence compared to preparation $B$, shows a significantly lower affinity for GPIb. In contrast, both preparations display an equal binding affinity for type III collagen. The relatively decreased function of preparation $A$ is confirmed by two sets of flow studies, investigating platelet adhesion and thrombus formation due to interaction of the GPIb-V-IX complex with VWF. High-shear flow experiments over type III collagen, using either reconstituted blood ${ }^{21}$ or whole blood from VWD patients, ${ }^{22}$ thus show significantly lower platelet deposition and thrombus formation in the presence of preparation A than of preparation $B$. Together, these finding suggest that the larger size VWF triplet bands, containing an additional $\mathrm{N}$-terminal peptide sequence, are more potent in recruiting platelets under arterial flow conditions. However, a limitation of our work is that the heparin fractionation had resulted in the partial loss of very high VWF multimers.

The presence or absence of the N-terminal fragment with GPIb binding site in the A1 domain may be considered 


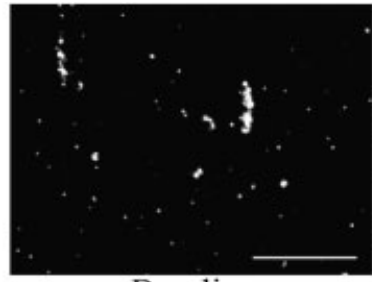

Baseline

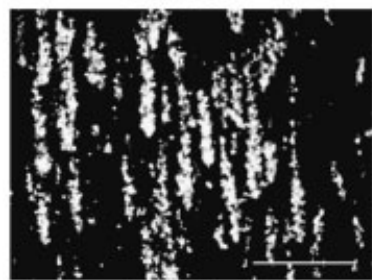

+ Prep. A

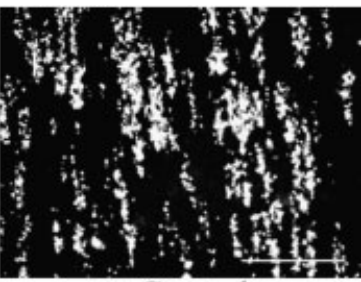

+ Control

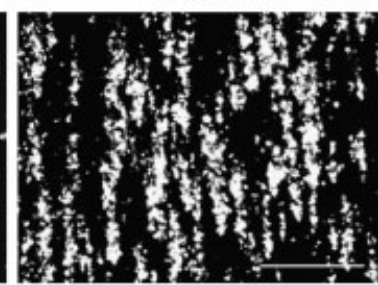

+ Prep. B

A

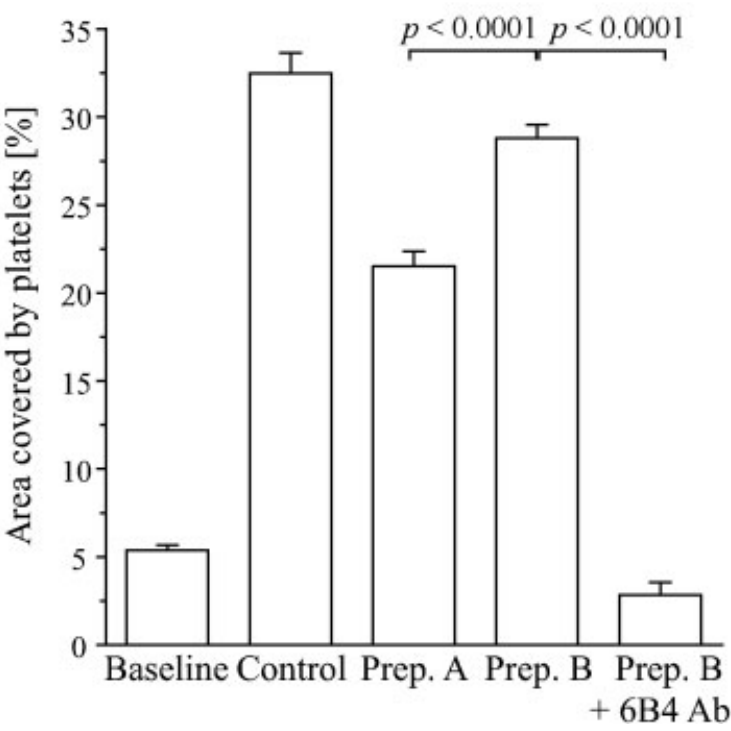

Fig. 6 Effect of VWF preparations on shear-dependent platelet adhesion in reconstituted blood. Flow chamber experiments performed with reconstituted blood containing CMFDA-labelled platelets $\left(2.5 \times 10^{8} / \mathrm{mL}\right)$ and washed red blood cells ( $40 \%$ hematocrit), flowed over a type III collagen surface at $1700 \mathrm{~s}^{-1}$ shear rate. Reconstituted blood samples were supplemented with control VWF preparation or preparation $A$ or $B(1 \mathrm{IU} / \mathrm{mL})$. The $6 \mathrm{~B} 4 \mathrm{mAb}(20 \mu \mathrm{g} / \mathrm{mL})$ against the VWF binding site of GPIb was added, as indicated. Samples without VWF (baseline) served as negative control.

(A) Representative fluorescence images (bars, $50 \mu \mathrm{m}$ ) after 8-minute perfusion. (B) Analyzed surface area coverage of fluorescence after 8-minute perfusion. Mean $\pm \operatorname{SEM}(n=6 ; 3$ different platelet preparations). CMFDA, 5-chloromethylfluorescein diacetate; GPIb, glycoprotein Ib; SEM, standard error of mean; VWF, von Willebrand factor.

theoretically negligible for platelet recruitment, taking into account the overall sum of other GPIb binding sites present in VWF multimers. However, given the present results, it is well feasible that this one additional GPIb binding site, located at the outer surface of globular multimers, has a considerable impact on protein function with regard to its sensitivity to conformational transition in response to fluid dynamic conditions and mechanical forces. Others have also reported on functional consequences due to the absence of the heparinbinding site within the A1 domain of VWF, following ADAMTS-13 cleavage. $^{32,33}$ The present results suggest that different functional properties of individual triplet bands are

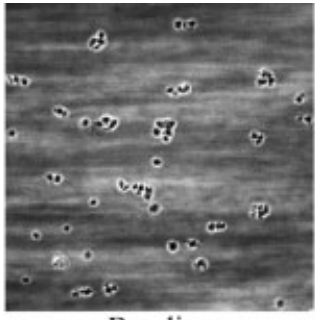

Baseline

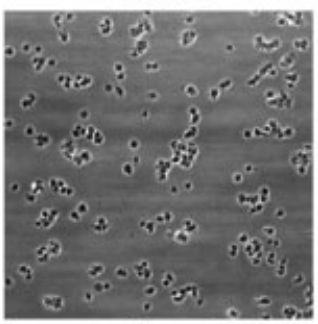

A

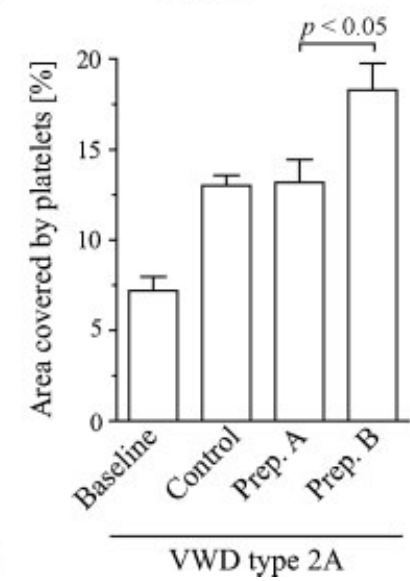

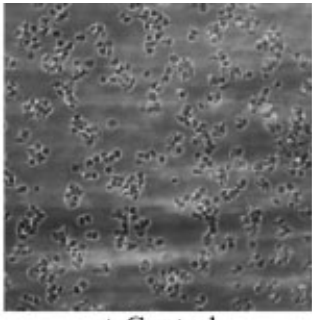

+ Control

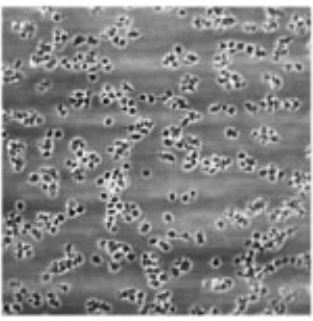

+ Prep. B

\section{B}

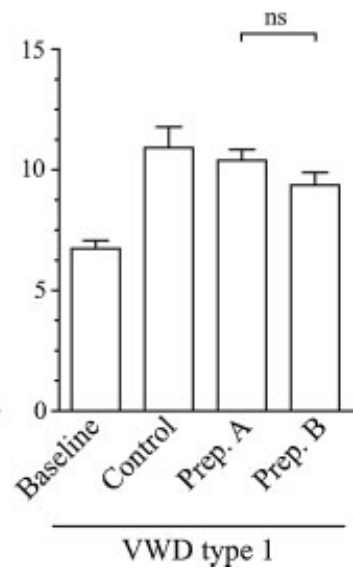

Fig. 7 Effect of VWF preparations on platelet adhesion added to blood from VWD patients. Whole blood from two VWD patients (type 1 VWD and $2 A$ VWD with $29 \%$ and $<10 \%$ VWF:RCo activity, respectively) was spiked with control VWF preparation or preparation A or B (2 IU/mL), and then perfused over collagen type III at a shear a rate of $1700 \mathrm{~s}^{-1}$. (A) Representative brightfield images after 4-minute perfusion of spiked blood from type 2 A patient $(150 \times 150 \mu \mathrm{m})$. (B) Analyzed surface area coverage of platelets after perfusion of spiked blood from type 2A VWD patient (left side) and type 1 VWD patient (right side). Mean \pm SEM ( $n=3$ independent experiments).

not only relevant for heparin affinity, but also for GPIb binding affinity.

Overall, the present results point to a distinct role of the VWF triplet band composition regarding GPIb-dependent platelet adhesion to VWF. The larger size, slower migrating VWF triplet bands appear to be functionally more active in supporting thrombus formation on collagen type III surfaces. The implication of these findings is that the altered triplet band composition regularly observed in type 2 VWD patients may contribute to an altered VWF-dependent platelet adhesion at high-shear flow.

\section{Acknowledgments}

We acknowledge support from the Cardiovascular Centre (HVC) Maastricht, the Netherlands Heart Foundation (2011T006) and the Center for Translational Molecular 
Medicine (CTMM) INCOAG, the Netherlands. We appreciate analytical support from Dr. A. Hillarp, (Malmö University Hospital, Sweden) and thank Dr. G. Kohla for critical review of the manuscript.

\section{Disclosure}

B. Fuchs, B. A. Solecka, M. Kröning, and C. Kannicht are employees of Octapharma R\&D, Molecular Biochemistry, Berlin. Other authors do not have disclosures.

\section{References}

1 Ruggeri ZM, Ruggeri ZM. Platelet and von Willebrand factor interactions at the vessel wall. Hamostaseologie 2004;24(1):1-11

2 Savage B, Saldívar E, Ruggeri ZM. Initiation of platelet adhesion by arrest onto fibrinogen or translocation on von Willebrand factor. Cell 1996;84(2):289-297

3 Hoylaerts MF, Yamamoto H, Nuyts K, Vreys I, Deckmyn H, Vermylen J. von Willebrand factor binds to native collagen VI primarily via its A1 domain. Biochem J 1997;324(Pt 1):185-191

4 Pareti FI, Niiya K, McPherson JM, Ruggeri ZM. Isolation and characterization of two domains of human von Willebrand factor that interact with fibrillar collagen types I and III. J Biol Chem 1987;262(28):13835-13841

5 Roth GJ, Titani K, Hoyer LW, Hickey MJ. Localization of binding sites within human von Willebrand factor for monomeric type III collagen. Biochemistry 1986;25(26):8357-8361

6 Cosemans JM, Schols SE, Stefanini L, et al. Key role of glycoprotein $\mathrm{Ib} / \mathrm{V} / \mathrm{IX}$ and von Willebrand factor in platelet activationdependent fibrin formation at low shear flow. Blood 2011;117 (2):651-660

7 Matsushita T, Dong Z, Sadler JE. von Willebrand's factor and von Willebrand's disease. Curr Opin Hematol 1994;1(5):362-368

8 Dong JF. Cleavage of ultra-large von Willebrand factor by ADAMTS13 under flow conditions. J Thromb Haemost 2005;3(8): $1710-1716$

9 Dent JA, Galbusera M, Ruggeri ZM. Heterogeneity of plasma von Willebrand factor multimers resulting from proteolysis of the constituent subunit. J Clin Invest 1991;88(3):774-782

10 Furlan M, Robles R, Affolter D, Meyer D, Baillod P, Lämmle B. Triplet structure of von Willebrand factor reflects proteolytic degradation of high molecular weight multimers. Proc Natl Acad Sci U S A 1993;90(16):7503-7507

11 Fischer BE, Thomas KB, Schlokat U, Dorner F. Triplet structure of human von Willebrand factor. Biochem J 1998;331(Pt 2):483-488

12 Fischer BE, Thomas KB, Schlokat U, Dorner F. Selectivity of von Willebrand factor triplet bands towards heparin binding supports structural model. Eur J Haematol 1999;62(3):169-173

13 Keuren JF, Ulrichts H, Feijge MA, et al. Integrin alphalIbbeta3 and shear-dependent action of glycoprotein Ibalpha stimulate plateletdependent thrombin formation in stirred plasma. J Lab Clin Med 2003;141(5):350-358

14 Neugebauer BM, Goy C, Budek I, Seitz R. Comparison of two von Willebrand factor collagen-binding assays with different binding affinities for low, medium, and high multimers of von Willebrand factor. Semin Thromb Hemost 2002;28(2):139-148

15 Budde U, Schneppenheim R, Plendl H, Dent J, Ruggeri ZM, Zimmerman TS. Luminographic detection of von Willebrand factor multimers in agarose gels and on nitrocellulose membranes. Thromb Haemost 1990;63(2):312-315

16 Matsubara Y, Murata M, Sugita K, Ikeda Y. Identification of a novel point mutation in platelet glycoprotein Ibalpha, Gly to Ser at residue 233 , in a Japanese family with platelet-type von Willebrand disease. J Thromb Haemost 2003;1(10):2198-2205

17 Russell SD, Roth GJ. Pseudo-von Willebrand disease: a mutation in the platelet glycoprotein Ib alpha gene associated with a hyperactive surface receptor. Blood 1993;81(7):1787-1791

18 Schneppenheim R, Obser T, Budde U, Patzke J. Development of a new functional assay for von Willebrand factor binding to platelet GpIba that does not require Ristocetin. Abstract presented at: Joint Meeting GTH \& NVTH; February 24-27, 2010; Nuremberg, Germany

19 Li F, Moake JL, McIntire LV. Characterization of von Willebrand factor interaction with collagens in real time using surface plasmon resonance. Ann Biomed Eng 2002;30(9):1107-1116

20 Romijn RA, Westein E, Bouma B, et al. Mapping the collagenbinding site in the von Willebrand factor-A3 domain. J Biol Chem 2003;278(17):15035-15039

21 Fuchs B, Budde U, Schulz A, Kessler CM, Fisseau C, Kannicht C. Flow-based measurements of von Willebrand factor (VWF) function: binding to collagen and platelet adhesion under physiological shear rate. Thromb Res 2010;125(3):239-245

22 Kuijpers MJ, Schulte V, Bergmeier W, et al. Complementary roles of glycoprotein VI and alpha2beta1 integrin in collagen-induced thrombus formation in flowing whole blood ex vivo. FASEB J 2003;17(6):685-687

23 Munnix IC, Kuijpers MJ, Auger J, et al. Segregation of platelet aggregatory and procoagulant microdomains in thrombus formation: regulation by transient integrin activation. Arterioscler Thromb Vasc Biol 2007;27(11):2484-2490

24 Munnix IC, Gilio K, Siljander PR, et al. Collagen-mimetic peptides mediate flow-dependent thrombus formation by high- or lowaffinity binding of integrin alpha2beta1 and glycoprotein VI. J Thromb Haemost 2008;6(12):2132-2142

25 De Meyer SF, Vanhoorelbeke K, Ulrichts H, et al. Development of monoclonal antibodies that inhibit platelet adhesion or aggregation as potential anti-thrombotic drugs. Cardiovasc Hematol Disord Drug Targets 2006;6(3):191-207

26 Budde U, Pieconka A, Will K, Schneppenheim R. Laboratory testing for von Willebrand disease: contribution of multimer analysis to diagnosis and classification. Semin Thromb Hemost 2006;32 (5):514-521

27 Furlan M, Robles R, Lämmle B. Partial purification and characterization of a protease from human plasma cleaving von Willebrand factor to fragments produced by in vivo proteolysis. Blood 1996;87 (10):4223-4234

28 Ruggeri ZM. Von Willebrand factor: looking back and looking forward. Thromb Haemost 2007;98(1):55-62

29 Michiels JJ, Gadisseur A, Budde U, et al. Characterization, classification, and treatment of von Willebrand diseases: a critical appraisal of the literature and personal experiences. Semin Thromb Hemost 2005;31(5):577-601

30 Michiels JJ, Berneman Z, Gadisseur A, et al. Classification and characterization of hereditary types $2 \mathrm{~A}, 2 \mathrm{~B}, 2 \mathrm{C}, 2 \mathrm{D}, 2 \mathrm{E}, 2 \mathrm{M}, 2 \mathrm{~N}$, and $2 \mathrm{U}$ (unclassifiable) von Willebrand disease. Clin Appl Thromb Hemost 2006;12(4):397-420

31 Studt JD, Budde U, Schneppenheim R, et al. Quantification and facilitated comparison of von Willebrand factor multimer patterns by densitometry. Am J Clin Pathol 2001;116(4):567-574

32 Mohri H, Fujimura Y, Shima M, et al. Structure of the von Willebrand factor domain interacting with glycoprotein Ib. J Biol Chem 1988;263(34):17901-17904

33 Sobel M, Soler DF, Kermode JC, Harris RB. Localization and characterization of a heparin binding domain peptide of human von Willebrand factor. J Biol Chem 1992;267(13):8857-8862 